\title{
Understanding Price Controls and Nonprice Competition with Matching Theory
}

\author{
By John William Hatfield, Charles R. Plott, and Tomomi Tanaka*
}

Price controls are one of the most common forms of market intervention by regulatory authorities. Price setting was a central duty of the Civil Aeronautics Board until the Airline Deregulation Act of 1978. The Dairy Price Support Program sets a minimum price for milk and other dairy products in the United States. Price caps for pharmaceutical drugs are a pervasive form of government intervention in Europe. And minimum wage laws set a price floor for labor throughout the developed world.

A great deal of empirical work suggests that price controls induce quality-based competition between sellers. ${ }^{1}$ However, competitive equilibria often fail to exist when price controls are present, and hence theoretical analysis of the effects of price controls has proven difficult. In particular, this lack of a theoretical foundation

\footnotetext{
* Hatfield: Graduate School of Business, Stanford University, 655 Knight Way, Stanford, CA 94305 (e-mail: hatfield@stanford.edu); Plott: Division of the Humanities and Social Sciences, California Institute of Technology, 1200 East California Boulevard, Pasadena, CA 91125 (e-mail: cplott@hss.caltech.edu); Tanaka: School of Politics and Global Studies, Arizona State University, P.O. Box 873902, Tempe, AZ 85287 (e-mail: tomomi.tanaka@asu.edu). We thank Marcus Berliant, Clayton Featherstone, Scott Duke Kominers, Muriel Niederle, Alvin E. Roth, Nilanjan Roy, William Zame, and seminar participants at Boston College, the 2009 Lee Center Workshop at Caltech, Harvard Business School, and New York University for helpful discussions. The financial support of the Gordon and Betty Moore Foundation and the support of the Caltech Laboratory of Experimental Economics and Political Science are gratefully acknowledged. Hatfield appreciates the hospitality of University of Tokyo, which hosted them during parts of this research.

${ }^{\dagger}$ To view additional materials, visit the article page at http://dx.doi.org/10.1257/aer.102.3.371.

${ }^{1}$ See Plott (1965) for an analysis of nonprice competition by regulated dry cleaners, Douglas and Miller III (1974) for an analysis of nonprice competition by airlines, and Joskow (1980) for an analysis of nonprice competition by hospitals. Hashimoto (1982) and Neumark and Wascher (2001) investigate whether minimum wage laws affect job characteristics, particularly on-the-job training.
}

undermines our ability to understand how price controls may induce changes in product quality.

As an example, consider the case where there are two sellers and one buyer, and two qualities, $H$ and $L$. The buyer has unit demand, and values a high quality good at 11 , while valuing the low quality good at 8 . Each seller has unit supply, with the low cost seller having a cost of production of 1 for a low quality good and 6 for a high quality good, and the high cost seller having a cost of production of 2 for a low quality good and 7 for a high quality good. In any competitive equilibrium, the low cost seller sells to the buyer the low quality good at a price $p(L) \in[1,2]$, with the price of the (untraded) high quality good being in the interval $p(H) \in[p(L)+3, p(L)+5]$. However, if a price floor of 5 is imposed for all qualities, then no competitive equilibrium exists, as both sellers will demand to sell since the price of the low quality good is at least 5 .

However, a stable outcome exists regardless of the existence of the price floor. In the case without the price floor, we know that any stable outcome induces the same allocation as the competitive equilibrium (Hatfield et al. 2012). When the price floor of 5 is present, our Theorem 3 demonstrates that in any stable outcome the low cost seller sells the high quality good to the buyer at a price in the interval $[6,7]$. This example demonstrates that a price floor may induce quality competition between sellers, and furthermore may reduce total social surplus. ${ }^{2}$

More generally, in this work we consider twosided markets with price controls as a special case of the two-sided matching with contracts model. ${ }^{3}$ Hatfield et al. (2012) showed that, when

\footnotetext{
${ }^{2}$ Indeed, in this example, all three agents are weakly worse off.

${ }^{3}$ Hatfield and Milgrom (2005) first developed the matching with contracts model, building on the work of Kelso and Crawford (1982); the possibility of such a generalization
} 
price controls are not present, the set of competitive equilibria naturally correspond to the set of stable outcomes. We develop a model of quality competition and show, using techniques developed by Echenique and Oviedo (2006) and Hatfield and Kominers (2012a, b), that the set of stable outcomes is nonempty even when price controls are imposed. Furthermore, the model exhibits quality-based competition induced by price controls: when a price floor is introduced, sellers compete by offering inefficiently high quality goods, while when a price ceiling is introduced, buyers compete by accepting inefficiently low quality goods.

\section{The Quality Competition Model}

\section{A. Framework}

There is a finite set of unit demand buyers $B$, a finite set of unit supply sellers $S$, and a finite set of qualities $Q$, where the set $Q$ is a finite set of consecutive integers $\left\{q^{\min }, \ldots, q^{\max }\right\}$. The set of trades is defined as $\Omega \equiv B \times S \times Q$. For each trade $(b, s, q)=\omega \in \Omega$, we let $\mathrm{b}(\omega) \equiv b$, $\mathrm{s}(\omega) \equiv s$, and $\mathrm{q}(\omega) \equiv q$. For a set of trades $\Psi \subseteq \Omega$, we let $\Psi_{b} \equiv\{\psi \in \Psi: b=\mathrm{b}(\psi)\}$ for each $b \in B$ and $\Psi_{s} \equiv\{\psi \in \Psi: s=\mathbf{s}(\psi)\}$ for each $s \in S$. A set of trades $\Psi \subseteq \Omega$ is feasible if each agent is associated with at most one trade, i.e., $\left|\Psi_{b}\right| \leq 1$ for all $b \in B$ and $\left|\Psi_{s}\right| \leq 1$ for all $s \in S$.

The set of contracts is defined as $X \equiv \Omega \times \mathbb{R}$. For a contract $x=\left(\omega, p_{\omega}\right)$, we let $\mathrm{b}(x) \equiv \mathrm{b}(\omega)$, $\mathrm{s}(x) \equiv \mathrm{s}(\omega), \mathrm{q}(x) \equiv \mathrm{q}(\omega)$, and $\mathrm{p}(x) \equiv p_{\omega}$. We let $Y_{b} \equiv\{y \in Y: b=\mathrm{b}(y)\}$ for each $b \in B$ and $Y_{s} \equiv\{y \in Y: s=\mathrm{s}(y)\}$ for each $s \in S$. A set of contracts $Y \subseteq X$ is an outcome if each agent is associated with at most one contract, i.e., $\left|Y_{b}\right| \leq 1$ for all $b \in B$ and $\left|Y_{s}\right| \leq 1$ for all $s \in S$.

The utility for a feasible set of trades for a buyer $b \in B$ is given by

$$
u^{b}(\Psi) \equiv \begin{cases}f^{b}+v(\mathbf{q}(\psi)) & \text { if } \exists \psi \in \Psi_{b} \\ 0 & \text { otherwise, }\end{cases}
$$

where $f^{b} \in \mathbb{R}$ denotes the value $b$ obtains from procuring a good, while $v(q)$ is a concave

was first noted in remarks by Crawford and Knoer (1981) and Kelso and Crawford (1982). Klaus and Walzl (2009) and Hatfield and Kominers (2012) extended this work to the setting of many-to many matching with contracts. function denoting the additional utility $b$ obtains from procuring a good of quality $q$. This induces a utility function over feasible outcomes for $b$ of

$$
U^{b}(Y) \equiv \begin{cases}f^{b}+v(\mathbf{q}(y))-\mathbf{p}(y) & \text { if } \exists y \in Y_{b} \\ 0 & \text { otherwise. }\end{cases}
$$

Similarly, the utility for a feasible set of trades for a seller $s$ is given by

$$
u^{s}(\Psi) \equiv \begin{cases}-c^{s}-e(\mathbf{q}(\psi)) & \text { if } \exists \psi \in \Psi_{s} \\ 0 & \text { otherwise, }\end{cases}
$$

where $c^{s} \in \mathbb{R}$ denotes the cost $s$ incurs from producing a good, while $e(q)$ is a convex function denoting the additional cost $s$ incurs from producing a good of quality $q$. This induces a utility function over feasible outcomes for $s$ of

$U^{s}(Y) \equiv \begin{cases}\mathrm{p}(y)-c^{s}-e(\mathrm{q}(y)) & \text { if } \exists y \in Y_{s} \\ 0 & \text { otherwise. }\end{cases}$

Finally, for simplicity, we assume that there is a unique quality $\hat{q}<q^{\max }$ that maximizes the surplus $v(q)-e(q)$; note that the assumptions on the utility function then imply that surplus is increasing with quality for all qualities below $\hat{q}$, and decreasing with quality for all qualities above $\hat{q}$.

\section{B. Efficiency and Equilibrium}

An outcome $A$ is efficient if it solves

$$
A \in \underset{Y \subseteq X}{\arg \max } \sum_{i \in B \cup S} U^{i}(Y) .
$$

It is straightforward that for any efficient outcome $A$, for each $x \in A, \mathrm{q}(x)=\hat{q}$.

The utility function for each agent induces a choice correspondence

$$
C^{i}(Y) \equiv \underset{Z \subseteq Y_{i}}{\arg \max } U^{i}(Z)
$$

for each $i \in B \cup S$.

We define an equilibrium as a stable outcome. An outcome $A \subseteq X$ is stable if it is

(i) Individually rational: for all $i \in B \cup S$, $A_{i} \in C^{i}(A)$. 
(ii) Unblocked: there does not exist a nonempty blocking set $Z \subseteq X$ such that

a) $Z \cap A=\varnothing$, and

b) for all $i \in B \cup S$, if $Z_{i} \neq \varnothing$, then $Z_{i} \subseteq Y^{i}$ for all $Y^{i} \in C^{i}(Z \cup A)$.

Stability is closely related to the idea of competitive equilibrium: Intuitively, for an allocation $A$ to be unblocked, for each contract $x \notin A$, it must be the case that either $\mathrm{b}(x)$ or $\mathrm{s}(x)$ rejects $x$. If so, for each agent $i$ we can consider his "budget set" as the union of $A_{i}$ and the set of contracts he rejects. Hence, stability insists that each agent chooses optimally from this "budget set," which is analogous to the notion of individual maximization from competitive equilibrium. Furthermore, market clearing is satisfied, as a contract $x$ is demanded by the buyer $\mathrm{b}(x)$ if and only if it is demanded by the seller $\mathrm{s}(x)$. Hatfield et al. (2012) make this notion precise, showing that a natural correspondence between competitive equilibria and stable outcomes exists in our setting when no price controls are imposed.

Furthermore, in this setting the set of stable outcomes is equivalent to the core. This equivalence no longer holds in settings with multiunit supply sellers and multiunit demand buyers.

\section{Equilibria of the Quality Competition Model}

We begin by considering the case where only contracts in $X(q) \equiv\{x \in X: \mathrm{q}(x)=q\}$ are available, but no price controls are present. In that case, $A$ is a stable outcome if and only if

$$
A \in \underset{Y \subseteq X(q)}{\arg \max } \sum_{i \in B \cup S} U^{i}(Y) ;
$$

that is, $A$ maximizes surplus given the contractual set available.

Furthermore, every contract will trade at the same price $p$. The price $p$ must be no less than

$$
p_{B}^{\min }(q) \equiv \max _{b \in B \backslash \mathrm{b}(Y)} f^{b}+v(q)
$$

so that each buyer with unmet demand does not wish to buy a good, and

$$
p_{S}^{\min }(q) \equiv \max _{s \in \mathbf{S}(Y)} c^{s}+e(q)
$$

so that each seller currently engaged in trade wishes to do so; we let $p^{\min }(q)$ $\equiv \max \left\{p_{B}^{\min }(q), p_{S}^{\min }(q)\right\}$. Analogously, the price $p$ must be no greater than

$$
p_{B}^{\max }(q) \equiv \min _{b \in \mathbf{b}(Y)} f^{b}+v(q)
$$

so that each buyer currently engaged in trade wishes to do so, and

$$
p_{S}^{\max }(q) \equiv \min _{s \in S \backslash \mathrm{s}(Y)} c^{s}+e(q)
$$

so that each seller not currently producing does not wish to supply a good; we let $p^{\max }(q)$ $\equiv \min \left\{p_{B}^{\max }(q), p_{S}^{\max }(q)\right\}$.

We formalize these results in Theorem 1; proofs of this and other stated results are a special case of results found in Hatfield et al. (2011).

THEOREM 1: For the contractual set $X(q)$, there exists at least one stable outcome A; for any such $A,(1)$ holds and moreover for each $x \in A, \mathrm{p}(x) \in\left[p^{\min }(q), p^{\max }(q)\right]$.

It is now straightforward to characterize the set of equilibria for the contractual set $X$.

THEOREM 2: For the contractual set $X$, the set of stable outcomes is the same as that given in Theorem 1 for the quality $\hat{q}$.

Hence, in the absence of price controls, any stable outcome will be efficient. In particular, only the efficient quality will trade. To see this, suppose that $y \in A$ for some outcome $A$ and that $\mathrm{q}(y) \neq \hat{q}$. Now consider $x$ such that $\mathrm{b}(x)=\mathrm{b}(y)$, $\mathbf{s}(x)=\mathbf{s}(y), \mathbf{q}(x)=\hat{q}$, and

$$
\mathbf{p}(x)=\mathbf{p}(y)+(v(\hat{q})-v(\mathbf{q}(y)))-\epsilon
$$

for some small $\epsilon>0$. Then $\{x\}$ is a blocking set, as $x$ will clearly be chosen by $\mathrm{b}(x)$, and also by $\mathrm{s}(x)$ since

$$
v(\hat{q})-v(\mathbf{q}(y))-\epsilon>c(\hat{q})-c(\mathbf{q}(y))
$$

for $\epsilon$ sufficiently small, as $\hat{q}$ is the unique efficient quality.

We now consider the case where a price floor of $p_{f}$ is imposed; that is, the set of contracts is 
given by $X\left[p_{f}\right] \equiv\left\{x \in X: \mathrm{p}(x) \geq p_{f}\right\}$. In this case, stable outcomes may no longer be efficient, as the supply of goods of quality $\hat{q}$ exceeds the demand. Hence, quality may adjust upward to compensate. When the price floor is just above $p^{\max }(\hat{q})$, trade of both the efficient quality $\hat{q}$ and the inefficient quality $\hat{q}+1$ may be observed; the efficient quality good will trade at the price floor $p_{f}$, while $\hat{q}+1$ quality goods will trade at $p_{f}+(v(\hat{q}+1)-v(\hat{q}))$. Trades of the inefficiently high quality good are not blocked at this price, as any blocking contract would have a price lower than the price floor. Finally, as the price floor $p_{f}$ rises above $p^{\max }(\hat{q}+1)-$ $(v(\hat{q}+1)-v(\hat{q}))$, we reach another tipping point, as all trade at the efficient quality ceases. At such high price floors, sellers compete away the gains from trading the efficient quality good by offering buyers more attractive contracts for the inefficient quality good; hence, only quality $\hat{q}+1$ goods will be traded in equilibrium.

THEOREM 3: Consider the contractual set $X\left[p_{f}\right]$. A stable outcome exists. There are three cases:

(i) $p_{f}<p^{\min }(\hat{q})$ : Then any stable outcome is as in Theorem 2.

(ii) $p^{\max }(\hat{q})<p_{f}<p^{\min }(\hat{q}+1)-[v(\hat{q}+$ 1) $-v(\hat{q})]:$ Then in any stable outcome $A$, for each $x \in A$ either

- $\mathrm{q}(x)=\hat{q}$ and $\mathrm{p}(x)=p_{f}$, or

- $\mathrm{q}(x)=\hat{q}+1 \quad$ and $\quad \mathrm{p}(x)=p_{f}+$ $(v(\hat{q}+1)-v(\hat{q}))$,

and the set of buyers engaging in trade is an element of

$$
\underset{\hat{B} \subseteq B}{\arg \max }\left\{\sum_{b \in \hat{B}}\left(f^{b}+v(\hat{q})-p_{f}\right)\right\} .
$$

(iii) $p^{\max }(\hat{q}+1)-[v(\hat{q}+1)-v(\hat{q})]<$ $p_{f}<p^{\min }(\hat{q}+1)$ : Then any stable outcome is as in Theorem 1 for the contract set $X(\hat{q}+1)$.

Price floors induce inefficiency in three ways: First, there is too little trade, since some welfare enhancing trades require a price below the price floor to be individually rational. Second, trade may be at an inefficient quality, since a mutually advantageous switch to a more efficient quality requires the price to drop below the price floor. Finally, the wrong agents may trade, as supply is greater than demand; a more efficient producer can not undercut a less efficient producer on price if the price is already at the price floor. ${ }^{4}$

\section{Conclusion}

The model presented here makes specific predictions of market equilibrium in settings with price controls. The results of our earlier work Hatfield, Plott, and Tanaka (2011) generalize the results here to the case of multiunit demand. Moreover, in that work, we report on a series of experiments of continuous time, double auction markets with multiple qualities; for each experiment, we impose a price control that corresponds to either part (ii) or part (iii) of Theorem 3. The experimental results are very close to the predictions of the theory: When the price floor falls within the regime of part (ii) of Theorem 3, agents trade both qualities, with the efficient quality good being traded at the price floor and the inefficiently high quality good being traded at a price reflecting a buyer's difference in valuation between the two qualities. When the price floor is higher, and falls within the regime of part (iii) of Theorem 3, agents trade only the high quality good and do so at a price as if that quality were the only quality available. Furthermore, in both cases, the quantity traded falls within the interval predicted by Theorem 3. Analogous results are obtained when price ceilings are imposed.

Stability is a robust solution concept which generalizes the concept of competitive equilibrium in settings with transferable utility. In this work, we show that stable outcomes exist even in settings with price controls, while competitive equilibria do not. This suggests that stability may be helpful in understanding the effects of other market interventions. For instance, in settings with quotas (such as import restrictions on certain goods), competitive equilibria fail to exist; however, stable outcomes do exist in such settings, and, hence, matching theory allows us

\footnotetext{
${ }^{4}$ The results of this theorem can be extended to any price floor, as well as to price ceilings. See Hatfield, Plott, and Tanaka (2011).
} 
to make sharp predictions. ${ }^{5}$ We conjecture that there may be other settings where stability provides sharp equilibrium predictions even though competitive equilibria fail to exist.

\section{REFERENCES}

-Crawford, Vincent P., and Elsie Marie Knoer. 1981. "Job Matching with Heterogeneous Firms and Workers." Econometrica 49 (2): 437-50.

Douglas, George W., and James C. Miller, III. 1974. "Quality Competition, Industry Equilibrium, and Efficiency in the Price-Constrained Airline Market." American Economic Review 64 (4): 657-69.

Echenique, Federico, and Jorge Oviedo. 2006. "A Theory of Stability in Many-to-Many Matching Markets." Theoretical Economics 1 (2): 233-73.

Hashimoto, Masanori. 1982. "Minimum Wage Effects on Training on the Job." American Economic Review 72 (5): 1070-87.

Hatfield, John William, and Scott Duke Kominers. 2012a. "Contract Design and Stability in Matching Markets." Unpublished.

-Hatfield, John William, and Scott Duke Kominers. 2012b. "Matching in Networks with Bilateral Contracts." American Economic Journal: Microeconomics 4(1): 176-208.

Hatfield, John William, and Paul R. Milgrom. 2005. "Matching with Contracts."
American Economic Review 95 (4): 913-35.

Hatfield, John William, Charles R. Plott, and Tomomi Tanaka. 2011. "Price Controls, NonPrice Quality Competition, and the Nonexistence of Competitive Equilibrium." Unpublished.

Hatfield, John William, Scott Duke Kominers, Alexandru Nichifor, Michael Ostrovsky, and Alexander Westkamp. 2012. "Stability and Competitive Equilibrium in Trading Networks." Unpublished.

- Joskow, Paul L. 1980. "The Effects of Competition and Regulation on Hospital Bed Supply and the Reservation Quality of the Hospital." Bell Journal of Economics 11 (2): 421-47.

-Kelso, Alexander S., Jr., and Vincent P. Crawford. 1982. "Job Matching, Coalition Formation, and Gross Substitutes." Econometrica 50 (6): 1483-504.

-Klaus, Bettina, and Markus Walzl. 2009. "Stable Many-to-Many Matchings with Contracts." Journal of Mathematical Economics 45 (7-8): 422-34.

- Neumark, David, and William Wascher. 2001. "Minimum Wages and Training Revisited." Journal of Labor Economics 19 (3): 563-95.

-Ostrovsky, Michael. 2008. "Stability in Supply Chain Networks." American Economic Review 98 (3): 897-923.

-Plott, Charles R. 1965. "Occupational Self-Regulation: A Case Study of the Oklahoma Dry Cleaners." Journal of Law and Economics 8: 195-222.

\footnotetext{
${ }^{5}$ Using matching theory to model settings with quotas was first suggested by Ostrovsky (2008).
} 